\title{
Situación epidemiológica de la tuberculosis en Chile en el escenario global 2018
}

\author{
NADIA ESCOBAR S.*
}

\section{Epidemiological situation of tuberculosis in Chile, 2018}

The first High Level Meeting of the General Assembly of the United Nations on ending Tuberculosis was performed in September 2018. In the meeting, the Member States subscribed new commitments and goals for 2022. In Chile in 2017, the tuberculosis incidence rate increased to 15.6 cases per 100,000, with important heterogeneity within geographic regions. Specific tuberculosis mortality rate remains stable, reaching 1.2 per 100,000 in 2016, also with heterogeneity within regions. Among the cases diagnosed in 2017, the most frequent risk group was the elderly (21.4\% of cases), followed by foreign migrants (15.5\%). In the same year, 37 cases of TB-MDR/RR (multidrug-resistant and Rifampicin-monoresistant TB) were diagnosed, and this number has grown steadily during the last 5 years. Treatment outcomes of the 2016 new cases cohort are as follows: $78.9 \%$ completed treatment, $10.5 \%$ deceased, and $6.6 \%$ lost to follow-up. Results in previously treated patients, HIV-TB co-infected patients, and TB-MDR patients, are significantly worse. The household contacts investigation coverage was $86.4 \%$ in all age, and $91.8 \%$ in under 15 years old. Tuberculosis remains a relevant public health issue in Chile and globally. The international tuberculosis agenda was reactivated in 2018, and this involved the generation of new global commitments in order to accelerate the accomplishment of the global goals in the End TB Strategy.

Key words: Tuberculosis; Public Health; Incidence; Risk factors; HIV infections; Multidrugresistance; United Nations.

\section{Resumen}

La primera 'Reunión de Alto Nivel de la Asamblea General de las Naciones Unidas'sobre la lucha contra la tuberculosis se concretó en septiembre de 2018. En ella los Estados Miembros suscribieron compromisos con metas para el año 2022. En Chile, la incidencia de tuberculosis aumentó en 2017, alcanzando 15,6 casos por 100.000 habitantes, con amplia heterogeneidad entre regiones del país. La mortalidad especifica por tuberculosis se mantiene estable, alcanzando 1,2 por 100.000 en el año 2016, con importantes diferencias en distribución geográfica. Entre los casos de tuberculosis diagnosticados en 2017, el grupo vulnerable más frecuente fue el de adultos mayores $(21,4 \%)$, seguido de las personas extranjeras $(15,5 \%)$. El mismo año se diagnosticaron 37 casos de TB-MDR/RR (multidrogo-resistente y mono resistente a rifampicina), cifra en crecimiento durante el último quinquenio. El índice de pesquisa fue de 22,6, levemente superior al año anterior. Los resultados de la cohorte de tratamiento 2016 para casos nuevos fueron: 78,9\% altas, 10,5\% fallecidos y 6,6\% pérdida de seguimiento. Las cohortes de pacientes antes tratados, coinfectados TB-VIH y pacientes con TB-MDR tienen resultados significativamente peores. Finalmente, la cobertura de estudios de contactos en 2017 fue de 86,4\% en general y $91,8 \%$ en contactos menores de 15 años. La tuberculosis es un importante problema de salud pública en Chile y el mundo. En 2018 se ha reactivado la agenda internacional de la lucha contra la tuberculosis y se han adquirido nuevos compromisos para acelerar los avances hacia las metas de la Estrategia Fin a la Tuberculosis.

Palabras clave: Tuberculosis; Salud Pública; Incidencia; Factores de riesgo ; Infecciones VIH; Multidrogo-resistencia; Naciones Unidas.

\footnotetext{
* Directora de Programa de Control y Eliminación de la Tuberculosis, Ministerio de Salud, Chile.
} 


\section{Introducción}

El 26 de septiembre de 2018 en Nueva York se realizó la primera 'Reunión de Alto Nivel de la Asamblea General de las Naciones Unidas' sobre la lucha contra la tuberculosis. En esta ocasión histórica participaron más de 40 Estados Miembros, representados por jefes de Estado, ministros de salud y parlamentarios, además de representantes de organizaciones de la sociedad civil y agencias donantes ${ }^{1}$. La reunión concluyó con una Declaración Política que reafirma el compromiso de alcanzar las metas de la 'Estrategia Fin a la Tuberculosis' para 2035, y establece nuevos compromisos con metas globales a 2022, entre ellos tratar con éxito a 40 millones de enfermos de tuberculosis entre 2018 y 2022, tratar la infección latente en 30 millones de personas para el 2022, así como movilizar financiamiento sostenible para servicios e investigación ${ }^{2}$.

En este contexto, la 'Alianza Fin a la Tuberculosis' estima que para cumplir con los compromisos firmados en la declaración, Chile debe diagnosticar y tratar al menos 14.900 casos entre 2018 y $2022^{3}$. Esta estimación es congruente con la evolución de la epidemia en el país observada en los últimos dos años, que muestra un alza significativa de la incidencia de tuberculosis en todas sus formas, y particularmente de la tuberculosis pulmonar con confirmación bacteriológica ${ }^{4}$.

El 'Reporte Global de Tuberculosis' emitido cada año por la OMS estimó que en 2017 1,6 millones de personas murieron por tuberculosis. Entre ellos, 300.000 serían coinfectados con VIH. Además se estima que para el mismo año, 10 millones de personas enfermaron de tuberculosis en el mundo, lo que incluye a un millón de niños. Por otra parte, se estima que 558.000 casos correspondieron a tuberculosis resistente a rifampicina (con o sin resistencia a isoniacida), alcanzando globalmente un 3,5\% de los casos nuevos y un $18 \%$ de los antes tratados 5 .

Este artículo detalla la situación epidemiológica actual de la tuberculosis en Chile, y analiza estos datos en el contexto de las metas globales establecidas en la 'Reunión de Alto Nivel de la Asamblea General de Naciones Unidas'. Finalmente, se discuten los principales desafíos que enfrenta hoy el Programa Nacional de Control y Eliminación de la Tuberculosis.

\section{Situación epidemiológica de la tuberculosis en Chile}

\section{Mortalidad}

La tasa de mortalidad específica por tuberculosis para el año 2016 fue de 1,2 por 100.000 habitantes, cifra que ha mostrado una tendencia leve a la disminución en la última década. La Figura 1 muestra la tasa de mortalidad por tuberculosis en Chile entre 2006 y $2016^{6}$.

\section{Incidencia}

La tasa de incidencia de tuberculosis fue de 15,6 casos por 100.000 habitantes para el año 2017 , lo que corresponde a un total de 2.740 casos, de acuerdo a la definición de incidencia de la Organización Mundial de la Salud 5 .

La Figura 2 muestra la evolución de la tasa de incidencia de tuberculosis en Chile desde el año 2006. Se observa que, luego de un período de desaceleración de la disminución de la incidencia de tuberculosis en el país, se revierte la tendencia $\mathrm{y}$ se produce un incremento en la tasa, alcanzando cifras similares a las de hace más de una década.

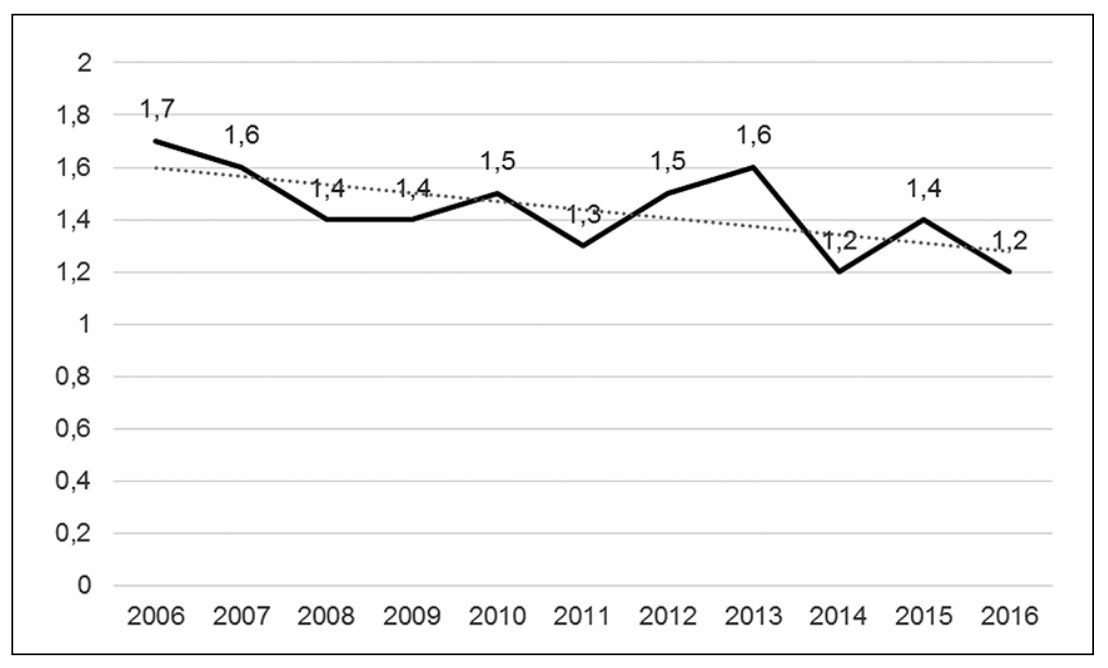

Figura 1. Tasa de mortalidad específica por tuberculosis en Chile 2006-2016. Fuente: Dirección de Estadística e Información de Salud (DEIS) 2017. Ministerio de Salud. Chile. 
El $81,1 \%$ de los casos corresponden a formas pulmonares, de los cuales el $91,3 \%$ cuenta con confirmación bacteriológica. La tasa de incidencia de tuberculosis pulmonar con bacteriología positiva en Chile el año 2017 fue de 11,5 por 100.000 habitantes, mostrando una evolución similar a la tasa de incidencia total de tuberculosis, según lo observado en la Figura 3.

\section{Distribución por edad y sexo}

Respecto a la distribución de los casos en la población, cabe destacar que la población masculina tiene una tasa 1,9 veces mayor que la femenina, y el mayor número de casos se concentra entre los 25 y 64 años de edad. Respecto al riesgo específico de cada grupo poblacional, la tasa de incidencia más alta se encuentra en los hombres mayores de 65 años. La Figura 4 muestra la tasa de incidencia de tuberculosis por edad y sexo en Chile para el año 2017.

Durante el año 2017 se produjeron 40 casos de tuberculosis en menores de 15 años en Chile. Cabe destacar que el $32,5 \%$ de ellos tiene como factor de riesgo el ser contacto de otra persona con tuberculosis pulmonar o laríngea con bacteriología positiva. La Figura 5 muestra la evolución del número de casos y la tasa de tuberculosis en menores de 15 años en el último quinquenio en Chile.

La tendencia de la tuberculosis infantil en Chile ha sido a la disminución en el último quinquenio. Sin embargo, el aumento de la incidencia de tuberculosis pulmonar con confirmación bacteriológica y la importancia de la condición de

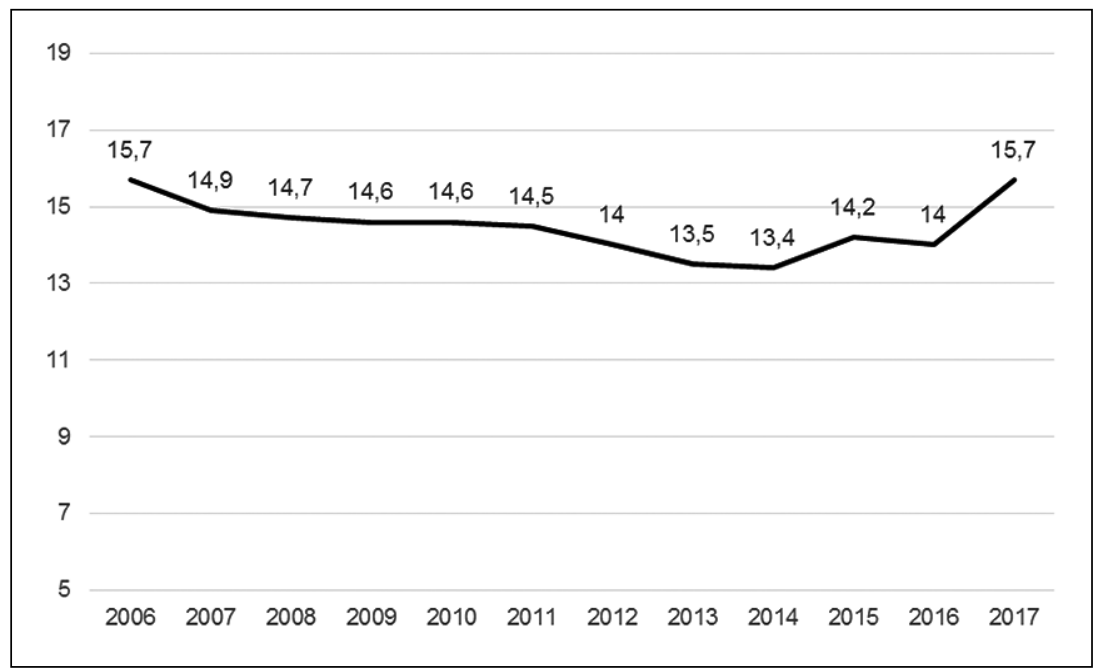

Figura 2. Tasa de incidencia de tuberculosis en Chile 2006-2017. Fuente: Registro Nacional del Programa de Tuberculosis.

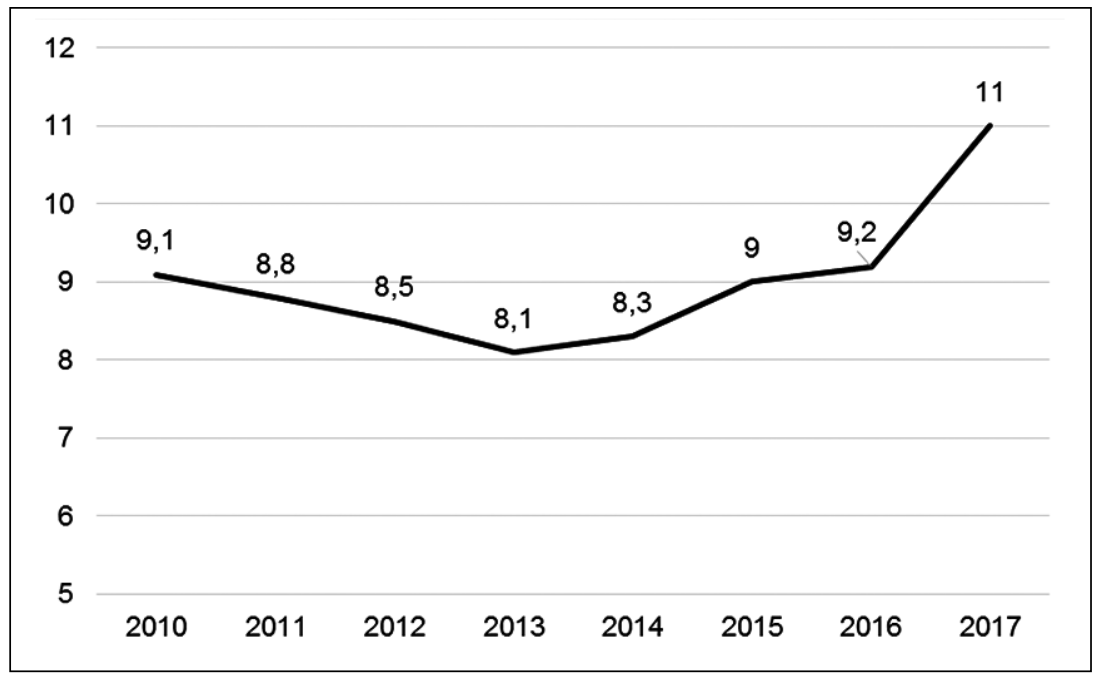

Figura 3. Tasa de incidencia de tuberculosis pulmonar con confirmación bacteriológica, Chile, 2010-2017. Fuente: Registro Nacional del Programa de Tuberculosis. 
contacto como factor de riesgo en la población infantil, permite predecir un probable incremento del número de casos infantiles de tuberculosis en Chile en los próximos años.

\section{Distribución geográfica}

La carga de tuberculosis se distribuye de manera heterogénea en el territorio nacional, como lo evidencian las tasas de incidencia y mortalidad diferenciadas por región. La mayor carga de enfermedad se encuentra en la macrozona norte. La tasa de incidencia más alta del país la presenta la región de Tarapacá (36 por 100.000 habitantes), seguida por las regiones de Arica y Parinacota $(27,9$ por 100.000$)$ y Antofagasta (23,9 por 1000.000$).{ }^{5}$ Las regiones con las tasas de mortalidad por tuberculosis más altas son
Tarapacá $(3,9$ por 100.000$)$, Los Ríos (3 por $100.000)$ y Arica y Parinacota $(2,5 \text { por } 100.000)^{6}$ (Figura 6).

\section{Poblaciones vulnerables}

De los grupos de la población identificados como vulnerables, el que se registra con mayor frecuencia entre los casos de tuberculosis es el de personas mayores a 65 años de edad, alcanzando un $21,4 \%$ del total de casos nuevos y recaídas. La Tabla 1 muestra la proporción de casos de tuberculosis de 2017 que pertenecen a cada grupo de riesgo. El 68,5\% de los casos 2017 tiene al menos un factor de riesgo.

La Figura 7 muestra la evolución de los grupos de riesgo más frecuentes en el último quinquenio, en porcentaje del total de casos.
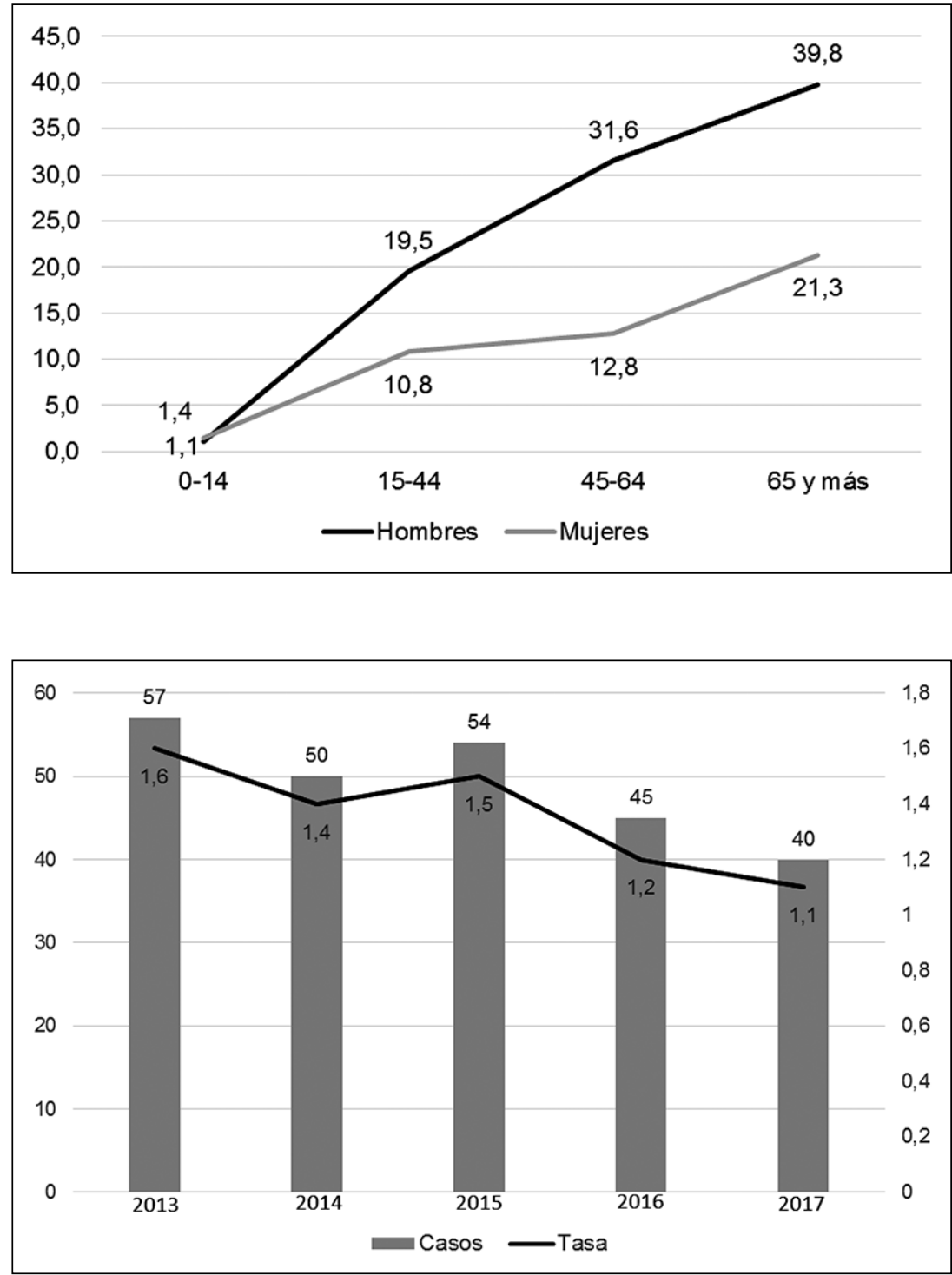

Figura 4. Tasa de incidencia de tuberculosis por edad y sexo, Chile año 2017. Fuente: Registro Nacional del Programa de Tuberculosis. 


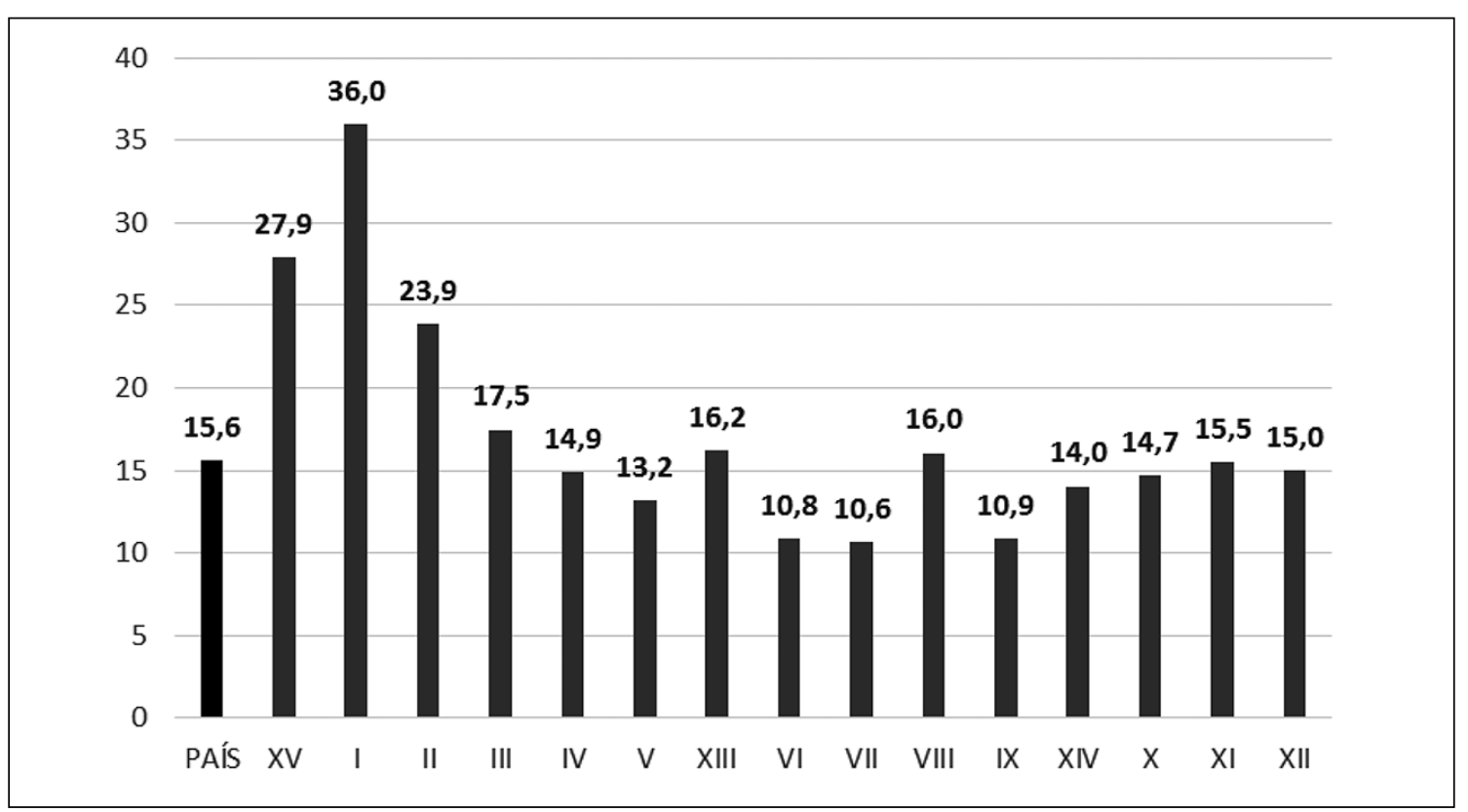

Figura 6. Tasa de incidencia de tuberculosis por región, Chile 2017. Fuente: Registro Nacional del Programa de Tuberculosis.

Tabla 1. Proporción de casos de tuberculosis por grupos de riesgo. Chile 2017

\begin{tabular}{|lc|}
\hline Grupo de riesgo & Proporción (\%) \\
\hline Adulto mayor & 21,4 \\
\hline Extranjeros & 15,5 \\
\hline Alcoholismo & 11,4 \\
\hline Drogadicción & 10,1 \\
\hline Co-infección VIH & 9,6 \\
\hline Diabetes & 7,4 \\
\hline Situación de calle & 4,7 \\
\hline Contactos & 3,7 \\
\hline Otra inmunosupresión & 2,9 \\
\hline Población privada de libertad & 2,6 \\
\hline Pueblo indígena & 2,5 \\
\hline Personal de salud & 1,1 \\
\hline
\end{tabular}

Fuente: Registro Nacional del Programa de Tuberculosis.

La población extranjera ha aumentado progresivamente su representación, de un $8,4 \%$ en 2013 a un $15,5 \%$ de los casos en 2017 . Aunque la cobertura de test VIH a los casos de tuberculosis se ha incrementado con los años, el porcentaje de coinfección TB-VIH se mantiene estable por debajo del $10 \%$, lo que es similar a la estimación global de $\mathrm{OMS}^{5}$. El reporte de diabetes en los casos de tuberculosis ha incrementado, iniciando el quinquenio con un $3,4 \%$ de los casos, a un
$7,4 \%$ de los casos en 2017. Si bien no se cuenta con los datos quinquenales para alcoholismo y drogadicción, los datos desde 2015 muestran un incremento en la frecuencia de ambos factores entre los casos de tuberculosis.

\section{Tuberculosis resistente a fármacos}

La tuberculosis multidrogorresistente y monorresistente a rifampicina (TB-MDR/RR) ha sido definida por la OMS como una crisis de la salud pública global, y este concepto se ratificó en la declaración política emanada de la 'Reunión de Alto Nivel de la Asamblea General de las Naciones Unidas' para poner fin a la tuberculosis. Mediante esta declaración, nuestro país suscribió al compromiso de mejorar el diagnóstico y los resultados de tratamiento de las personas que padecen TB-MDR/RR. Los países firmantes se comprometieron a diagnosticar y tratar a 1,5 millones de personas con tuberculosis resistente a fármacos entre 2018 y $2022 .^{2}$ De acuerdo a las estimaciones de la Alianza Fin a la Tuberculosis basadas en este compromiso, en Chile se deberían diagnosticar 37 personas con TB-MDR en el año 2019, 56 en el 2020, 96 en 2021 y 105 en $2022^{3}$.

Entre los casos nuevos diagnosticados el 2017 en Chile se encontraron 18 casos de TB-MDR y 13 casos de TB-RR. Entre los casos antes tratados ingresados en 2017 (recaídas y luego de pérdida de seguimiento), se diagnosticaron 5 TB-RR y 1 TB-MDR. 


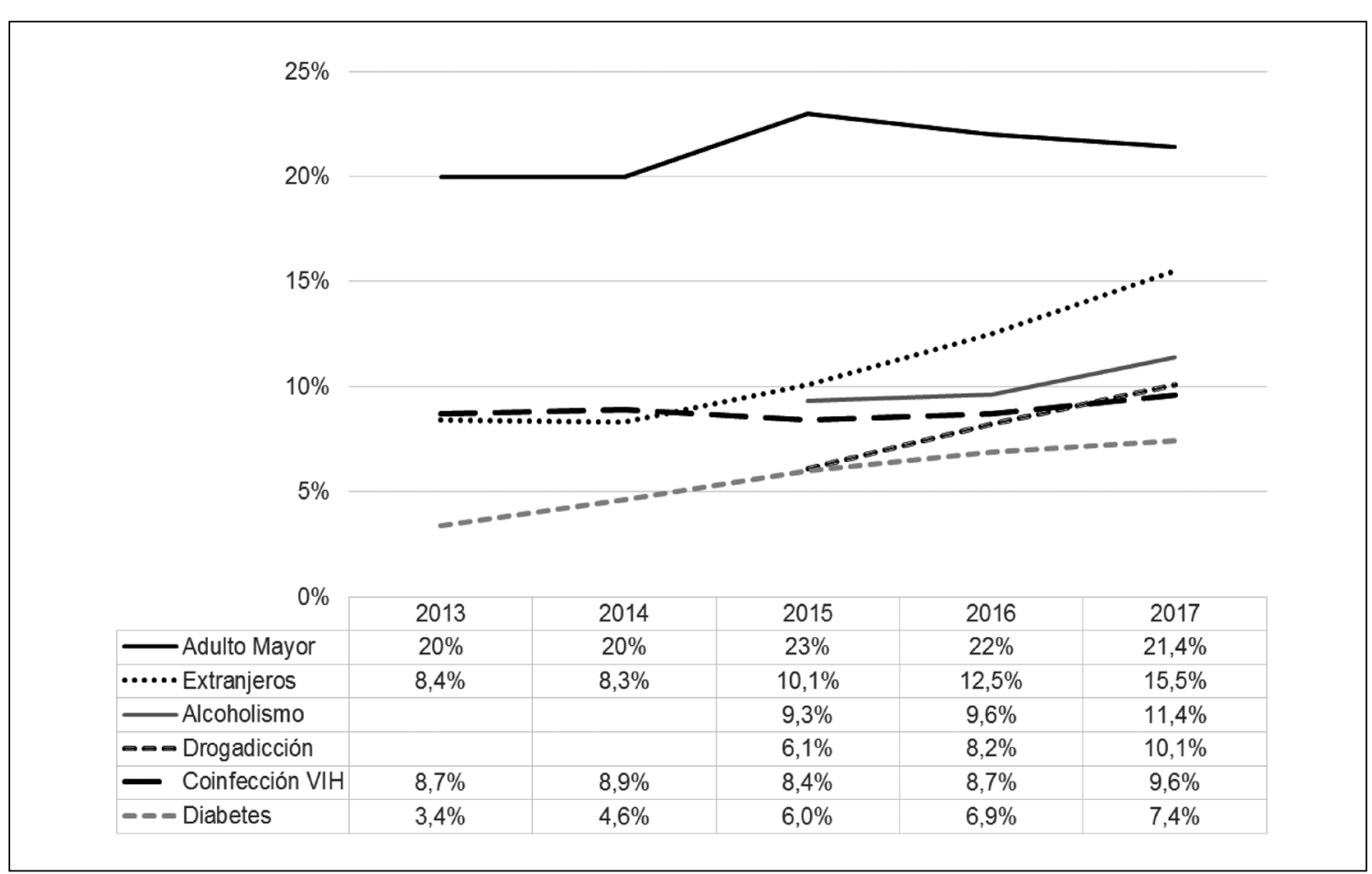

Figura 7. Proporción de casos de tuberculosis con los factores de riesgo más frecuentes en Chile quinquenio 2013-2017. Fuente: Registro Nacional del Programa de Tuberculosis.

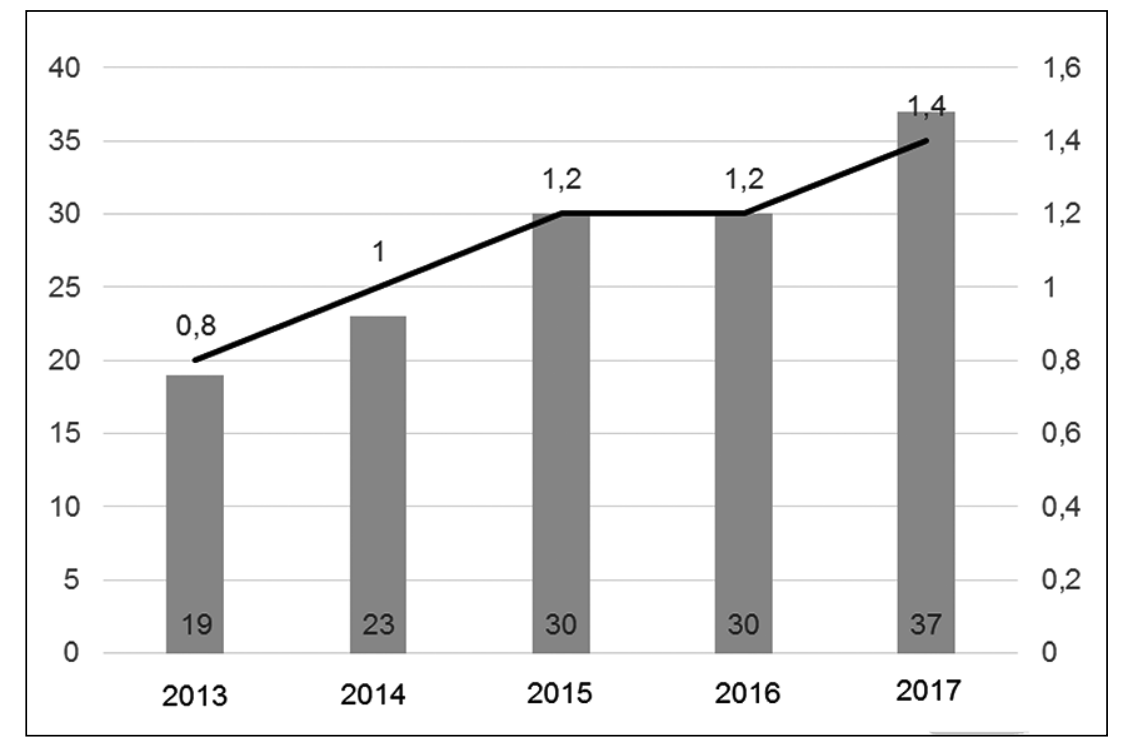

Figura 8. Número de casos de tuberculosis MDR/RR diagnosticados en Chile por año, $\mathrm{y}$ porcentaje del total de casos, 2013-2017. Fuente: Registro Nacional del Programa de Tuberculosis.
La Figura 8 muestra el número de casos TBMDR/RR diagnosticados en Chile durante el último quinquenio, y el porcentaje que representan del total de casos de ese año. Como se aprecia, tanto el número como la proporción de casos de tuberculosis MDR/RR se ha incrementado en el tiempo. Parte de este aumento es atribuible a la incorporación de la vigilancia universal de resistencia a drogas de primera línea, implementada en Chile desde el año 20147. Cabe destacar que estos casos siguen representando una pequeña proporción. Sin embargo, por su relevancia epidemiológica y clínica, concentran la dedicación de recursos y actividades. 


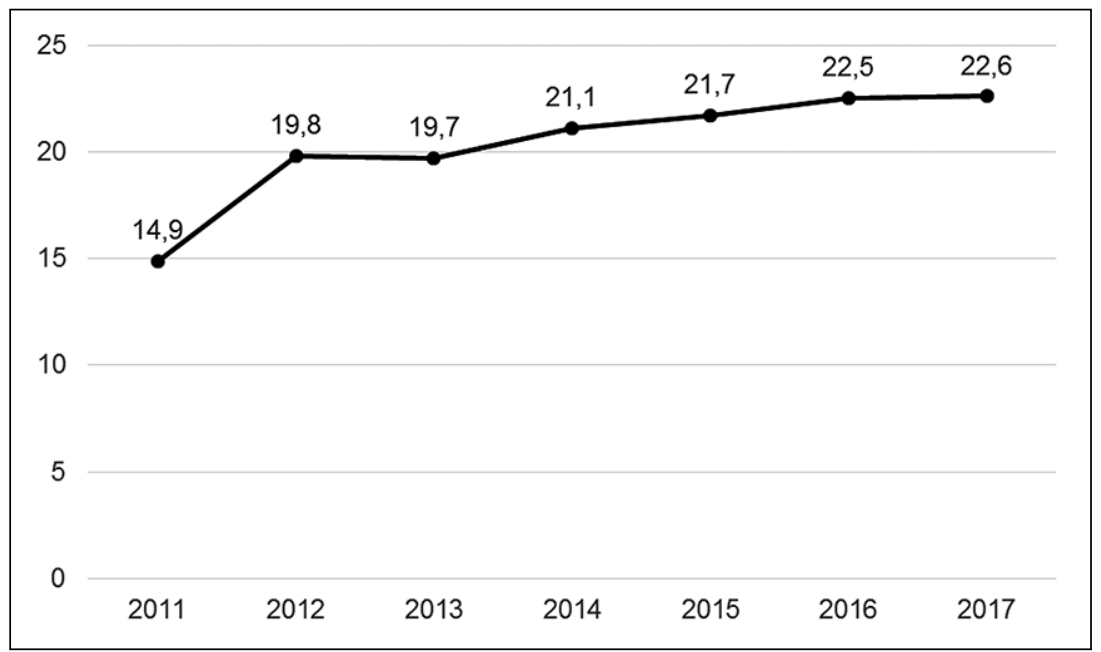

Figura 9. Índice de pesquisa de tuberculosis en Chile 2011-2017. Fuente: Acciones bacteriológicas de la red de laboratorios de tuberculosis. Instituto de Salud Pública (ISP).

\section{Resultados operacionales del programa}

\section{Localización de casos}

El Índice de Pesquisa (IP) relaciona el número de baciloscopías de diagnóstico procesadas con el total de consultas de morbilidad de personas mayores de 15 años. Para el año 2017, el IP a nivel nacional fue de 22,6. Si bien el índice ha aumentado en el tiempo a nivel nacional, persisten desafíos para mejorar el rendimiento de las estrategias mediante la focalización en grupos vulnerables. En la Figura 9 se presenta la evolución del IP nacional en el último quinquenio.

\section{Evaluación del tratamiento}

Las metas del programa son que al menos un $90 \%$ de los pacientes que ingresa a tratamiento primario sea dado de alta con su tratamiento terminado, con o sin confirmación bacteriológica de curación; que las pérdidas de seguimiento sean menores al 5\%; los fallecidos durante el tratamiento sean menos del 3\% (independiente de la causa); y que menos del $1 \%$ presente un fracaso de tratamiento.

Entre las personas que ingresaron a tratamiento primario en el año 2016 en Chile como casos nuevos, el 78,9\% logró un alta exitosa, el 10,5\% falleció durante el tratamiento, el $6,6 \%$ de los casos resultó en pérdida de seguimiento, y sólo se registró un caso con fracaso de tratamiento.

Entre los pacientes antes tratados para el mismo año, las altas del nuevo tratamiento sólo alcanzan el 57,4\%. La cohorte específica de los pacientes con coinfección VIH ingresados a tratamiento primario de tuberculosis el 2016 tuvo un $54,5 \%$ de altas. Por otra parte, los casos con TB-MDR ingresados a tratamiento de segunda línea en el año 2015 en Chile lograron solamente $43,8 \%$ de altas.

\section{Cobertura del estudio de contactos}

Durante el año 2017 se observó una leve mejoría de la cobertura de estudios de contactos en la red asistencial del país, alcanzando un $86,4 \%$ en general, y un $91,8 \%$ en los contactos menores de 15 años. Cabe mencionar que las metas programáticas de la cobertura del estudio de contactos son de $90 \%$ y $100 \%$ respectivamente, por lo que aún no se cumplen los objetivos.

\section{Conclusiones y desafíos}

El hecho que el año 2018 se haya intensificado la atención de los organismos internacionales sobre la epidemia de la tuberculosis no es una casualidad. La evolución más reciente de la epidemia ha demostrado que los avances alcanzados desde el comienzo de la 'Estrategia Fin a la Tuberculosis' son insuficientes. Mientras que globalmente la incidencia de tuberculosis disminuye discretamente, la brecha de casos no pesquisados se mantiene.

En la 'Reunión de Alto Nivel' de 2018 los países suscribieron al compromiso de tratar exitosamente a 40 millones de personas enfermas de tuberculosis para 2022. Para esto es indispensable hacer más accesible el diagnóstico en las poblaciones vulnerables, teniendo en cuenta que aproximadamente un tercio de los enfermos a nivel mundial no son diagnosticados. En Chile actualmente se diagnostica el $87 \%$ de la incidencia estimada, situación significativamente mejor que la global $^{8}$. Sin embargo, el desafío actual a 
nivel nacional radica en que las poblaciones más vulnerables accedan al diagnóstico oportunamente, para lo cual las estrategias intersectoriales resultan primordiales.

Adicionalmente, se requiere mejorar la tasa de éxito de tratamiento en el país. Si bien a nivel nacional la pérdida de seguimiento (también llamada abandono) alcanza el 6,6\% de la cohorte, en algunas regiones esa cifra se duplica ${ }^{4}$. Más aún, la proporción de fallecidos en la cohorte de tratamiento chilena es particularmente alta en comparación a otros países de la región ${ }^{9}$. Esto se puede explicar en parte por características demográficas. Sin embargo, también juega un papel el diagnóstico tardío de la enfermedad.

La tuberculosis infantil en Chile es infrecuente. Sin embargo, es esperable que en las condiciones epidemiológicas actuales aumente el número de casos anuales en menores de 15 años, teniendo en cuenta el aumento sostenido de la tasa de incidencia de tuberculosis pulmonar con bacteriología positiva. De forma similar, se espera un incremento en el número de casos con tuberculosis MDR/RR en Chile, de manera congruente con la epidemiología global, y también de acuerdo a un incremento en la capacidad diagnóstica de las resistencias.

Finalmente, si se busca eliminar la tuberculosis como problema de salud pública, no se puede desatender el diagnóstico y tratamiento de la infección latente. En este ámbito, el desafío para el Programa Nacional de Tuberculosis radica en modernizar los métodos diagnósticos y que estos sean accesibles para toda la red asistencial, además de incorporar nuevos esquemas terapéuticos que apoyen la adherencia y completitud de la terapia. En esto último, Chile ha avanzado implementando terapias más breves y de administración supervisada.

Lejos de ser un problema del pasado, la tuberculosis sigue siendo hoy un importante problema de salud pública en Chile y en el mundo. El escenario global justifica acciones dirigidas a acelerar los progresos de la 'Estrategia 'Fin a la Tuberculosis', y en nuestro país hay argumentos epidemiológicos suficientes para priorizar y reforzar la lucha contra la tuberculosis a través de la innovación y del abordaje integral de la epidemia en la comunidad.

\section{Bibliografía}

1.- ORGANIZACIÓN DE LAS NACIONES UNIDAS. Informe del Director General de la Asamblea General de las Naciones Unidas acerca de la Reunión de Alto
Nivel sobre la lucha contra la tuberculosis. Resolución A72/645. [En línea] Diciembre 2017. [Consultado el 19 de noviembre de 2018]. Disponible en: https://www. who.int/tb/features_archive/UNGA_report_on_HLMN TB.pdf?ua=1.

2.- ORGANIZACIÓN DE LAS NACIONES UNIDAS. Declaración política de la reunión de alto nivel de la Asamblea General de las Naciones Unidas sobre la lucha contra la tuberculosis. Resolución A73/3. [En línea] Octubre 2018. [Consultado el 19 de noviembre de 2018]. Disponible en: https://undocs.org/es/A/ RES/73/3.

3.- STOP TB PARTNERSHIP. UN High-Level Meeting (HLM) on TB, key targets and commitments for 2022, Key Targets per Country. [En línea] Octubre 2018. [Consultado el 19 de noviembre de 2018]. Disponible en: http://www.stoptb.org/global/advocacy/unhlm_targets.asp.

4.- PROGRAMA DE CONTROL Y ELIMINACIÓN DE LA TUBERCULOSIS. Informe de situación epidemiológica y operacional Chile 2017. Ministerio de Salud, Chile. Disponible en: https://diprece.minsal.cl/ wp-content/uploads/2018/10/2018.10.05_TUBERCULOSIS-2017.pdf.

5.- ORGANIZACIÓN MUNDIAL DE LA SALUD. Global Tuberculosis Report 2018. [En línea] 2018. [Consultado el 19 de noviembre de 2018]. Disponible en: https:// www.who.int/tb/publications/global_report/en/.

6.- DEPARTAMENTO DE ESTADÍSTICAS E INFORMACIÓN EN SALUD. Defunciones por algunas causas específicas de muerte según sexo, Serie Defunciones 2000-2016. Ministerio de Salud, Chile. [En línea] Disponible en: http://www.deis.cl/.

7.- HERRERA T. La tuberculosis en Chile: situación epidemiológica y avances del Programa Nacional de Control y Eliminación 2017. Rev Chil Enferm Respir 2017; 33 : 320-4.

8.- ORGANIZACIÓN MUNDIAL DE LA SALUD. Tuberculosis country profile: Chile 2017. [En línea] 2018. [Consultado el 19 de noviembre de 2018]. Disponible en: https:/www.who.int/tb/country/data/profiles/en/.

9.- ORGANIZACIÓN PANAMERICANA DE LA SALUD. Tuberculosis en las Américas 2018. [En línea] 2018. [Consultado el 19 de noviembre de 2018]. Disponible en: http://iris.paho.org/xmlui/ bitstream/handle/123456789/49510/OPSCDE18036 spa? sequence $=2 \&$ i.

Correspondencia a:

Dra. Nadia Escobar S.

Directora, Programa Nacional de Control y Eliminación de la Tuberculosis.

Ministerio de Salud, Gobierno de Chile.

Email: nadia.escobar@minsal.cl 